\title{
Three-User Gaussian Multiple Access Channel with Partially Cooperating Encoders
}

\author{
O. Simeone ${ }^{(1)}$, O. Somekh ${ }^{(2)}$, G. Kramer ${ }^{(3)}$, H. V. Poor ${ }^{(2)}$ and S. Shamai (Shitz) ${ }^{(4)}$ \\ ${ }^{(1)}$ CWCSPR, New Jersey Institute of Technology, Newark, NJ 07102, USA \\ ${ }^{(2)}$ Dept. of Electrical Engineering, Princeton University, Princeton, NJ 08544, USA \\ ${ }^{(3)}$ Bell Labs, Alcatel-Lucent, Murray Hill, NJ 07974, USA \\ ${ }^{(4)}$ Department of Electrical Engineering, Technion, Haifa, 32000, Israel.
}

\begin{abstract}
A three-user Gaussian multiple access channel (MAC) with encoders partially cooperating over a ring of finite-capacity uni-directional links is studied. The model is a simple extension of the classical two-user MAC with conferencing encoders. Upper and lower bounds on the maximum achievable common rate are derived. The lower bound is attained via a novel multi-layer cooperative strategy. The bounds are tight for a certain range of values of the inter-user link capacity. Numerical results are provided to corroborate the analysis.
\end{abstract}

\section{INTRODUCTION}

Complex networks in which terminals are potentially connected via a number of different radio interfaces or wired links are becoming increasingly appropriate models for current and future communication scenarios. In this paper, we focus on one such model in which three users are endowed each with two communication interfaces: (i) a radio transmitter to communicate with an access point via a Gaussian Multiple Access Channel (GMAC); and (ii) a radio or wired connection to "neighboring" users. More precisely, as sketched in Fig. 1, the users are connected to each other via a ring of finitecapacity uni-directional links, which can model either wired connections as in SONET/ SDH rings (see, e.g., [2]) or orthogonal wireless interfaces operated below their capacity. Each user is interested in transmitting data to an access point via the GMAC. The aim is obtaining a characterization of the maximum achievable equal rate per user.

\section{A. Related work}

The considered model is the simplest extension of the two-user multiple access channel (MAC) with partially cooperating, or conferencing, encoders introduced in [1], where the two users are connected via finite-capacity uni-directional links. Conferencing encoders have later been investigated in [3] for a two-user interference channel. These works show that conferencing encoders can exploit the inter-user links to create dependence between the signals transmitted on the main channels, thus leveraging multiple access "interference" for the purpose of "coherent combining". Conferencing encoders have also been studied in the context of source coding problems, namely for direct [4] [5], remote [6] and universal source

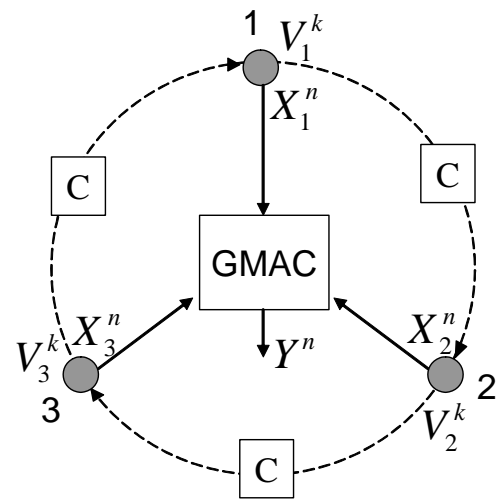

Fig. 1. Three users communicate with an access point over a GMAC, while being connected with each other via a ring of finite-capacity uni-directional links.

coding in [7] [8] $]^{1}$, all for a two-to-one scenario. Finally, conferencing decoders have also been considered, see, e.g., [9] [10] [11].

While previous work focused on two-user systems, here we consider a MAC with three users, which are connected via a ring of finite-capacity links (see Fig. 1). The problem of communicating over a ring of finite-capacity links has recently received attention per se in [12] [13], where sufficiency of routing (rather than network coding) to achieve capacity was proved for a class of multiple multicast sessions on undirected ${ }^{2}$ rings [12] and for multiple unicast sessions with bi-directional links in [13]. It is noted that here the goal is different from [12] [13] in that the value of inter-user links is in enabling cooperation over the GMAC rather than communicating among the users.

\section{B. Main contributions}

In this work, we focus on the system in Fig. 1 and consider as a performance criterion of interest the maximum equal rate achievable on the GMAC. It is noted that, while uni-directional

\footnotetext{
${ }^{1}$ In [4] [7], the signal over the finite-capacity links are also observed by the destination so that the model can also be interpreted in terms of feedback [7].

${ }^{2}$ With undirected links the sum of the traffic carried along both directions of a link is bounded by the capacity of the link.
} 
links are assumed throughout the text, some remarks on the scenario with undirected links are provided as well. We derive an upper bound on the equal rate and compare it with an achievable rate based on a novel multi-layer communication strategy. In this transmission scheme, the message of each user is split into different layers, some of which are then exchanged over the ring with a subset of the other users, thus creating common messages and the opportunity for cooperation. The strategy is an extension of the capacity-achieving scheme of [1] for the two-user case, and exploits the general capacity region for the MAC with common messages of [14]. We emphasize that, while for the two-user case optimality of this strategy was shown by [1], no extension of this result has been yet provided for a scenario with many users. Here we show that the strategy is capacity-achieving for large enough ring capacity $C$, and numerical results are provided to corroborate the analysis.

Notation: We write $[x]=[x-1]_{3}+1$, where $[\cdot]_{3}$ represents the mod-3 operation; $X_{\mathcal{S}}$ represents the collection of variables $X_{j}$ indexed by $j \in \mathcal{S}$ (i.e., $X_{\mathcal{S}}=\left\{X_{j}\right\}_{j \in \mathcal{S}}$ ) and, similarly, $X_{\mathcal{S}, i}=\left\{X_{j, i}\right\}_{j \in \mathcal{S}} ; X_{j}^{n}$ is the collection of variables $\left\{X_{j, i}\right\}_{i=1}^{n} ; \delta(x)$ is the Kronecker delta function; probability density functions (pdfs) are generally identified, except when explicitly stated otherwise, by their arguments, e.g., $p(x \mid y)$ is the conditional pdf of random variable $X$ given another random variable $Y$.

\section{SYSTEM MODEL}

We focus on the three-user GMAC in Fig. 1 where unidirectional (clockwise) links of finite-capacity $C$ (bit/ channel use) exist between adjacent users. On the GMAC, the received signal is given at the $i$ th symbol of a given encoding block $(i=1,2, \ldots, n)$ by

$$
Y_{i}=X_{1, i}+X_{2, i}+X_{3 . i}+N_{i},
$$

where the inputs have the same power constraint $\sum_{i=1}^{n} E\left[X_{j, i}^{2}\right] \leq n P, j=1,2,3$, and the Gaussian noise power is $E\left[N^{2}\right]=1$. Each $j$ th user generates a rate- $R$ message as $W_{j} \in\left\{1,2, \ldots, 2^{n R}\right\}$ per coding block intended for the destination. Coding over the ring of conferencing channels and the GMAC is modelled similarly to [1]. Specifically, we consider $k$ rounds of conferencing for each coding block. In every $i$ th round, a symbol $V_{j, i} \in \mathcal{V}_{j, i}$ is transmitted by the $j$ th user towards the $[j+1]$ th over the ring, which is a function $f_{j, i}(\cdot)$ of the local message $W_{j}$ and the symbols received during the previous rounds, i.e.,

$$
V_{j, i}=f_{j, i}\left(W_{j}, V_{[j-1]}^{i-1}\right) .
$$

Due to the capacity constraint, we also have the following cardinality bound: $\sum_{i=1}^{k} \log _{2}\left|\mathcal{V}_{j, i}\right| \leq n C$. Encoding on the GMAC takes place after the $k$ conferencing rounds as

$$
X_{j}^{n}=g_{j}\left(W_{j}, V_{[j-1]}^{k}\right),
$$

that is, the transmitted codeword is a function $g_{j}(\cdot)$ of both the local message and the symbols $V_{[j-1]}^{k}$ received during the $k$ conferencing rounds. It is noted that $R$ is the equal rate (in bit/channel use) and that definition of achievability is standard and based on the average probability of error $P_{e}=\operatorname{Pr}\left[\hat{W}_{\{1,2,3\}} \neq W_{\{1,2,3\}}\right]$, where $\hat{W}_{\{1,2,3\}}=\phi\left(Y^{n}\right)$ denotes the estimated messages at the receiver via the decoding function $\phi(\cdot)$ (see, e.g., [15]). The term capacity hereafter refers to the maximum achievable equal rate.

\section{AN UPPER BOUND}

An upper bound on the capacity of the system at hand is given by the following proposition.

Proposition 1: An upper bound on the capacity of the system in Fig. 1 is given by

$$
R_{\text {upper }}=\min \left\{R_{\text {upper }}^{\prime}, R_{\text {upper }}^{\prime \prime}\right\},
$$

with

$$
R_{\text {upper }}^{\prime}=\max _{0 \leq P^{\prime} \leq P} \min \left\{\begin{array}{c}
C+\frac{1}{6} \log \left(1+3 P^{\prime}\right), \\
\frac{1}{6} \log \left(1+9 P-6 P^{\prime}\right)
\end{array}\right\}
$$

and

$$
R_{\text {upper }}^{\prime \prime}=\max _{\substack{0 \leq P_{1} \leq 4 P, 0 \leq P_{2} \leq P}} \min \left\{\begin{array}{l}
\frac{1}{4} \log \left(1+P_{1}\right)+\frac{C}{2}, \\
\frac{1}{2} \log \left(1+P_{2}\right)+C, \\
\frac{1}{6} \log \left(1+P_{1}+P_{2}\right)+\frac{2}{3} C, \\
\frac{1}{6} \log (1+5 P \\
\left.+2 \sqrt{\left(4 P-P_{1}\right)\left(P-P_{2}\right)}\right)
\end{array}\right\} .
$$

Remark 1 (Sketch of proof of Proposition 1): The upper bound $R_{\text {upper }}^{\prime}$ (5) states that, having fixed power $P^{\prime}$ for noncooperative transmission, the capacity is upper bounded by the maximum sum-rate of a non-cooperative GMAC with an extra path of capacity $C$ from each source to the receiver (first term), and by the sum-rate achievable with full cooperation with the remain power $P=P^{\prime}$. Details of the proof can be found in Appendix-A. The second upper bound $R_{\text {upper }}^{\prime \prime}(6)$ is derived by considering a two-user GMAC with conferencing obtained as follows: enhance the original system in Fig. 1 by (i) assuming that one of the conferencing links, say between users 1 and 2, has unlimited capacity; and (ii) providing the message of user 2 to user 1 . As a result of these enhancements (which can only increase the capacity), we can see the system as made up of two users, one "regular" with power constraint $P$ and rate $R$ (user 3 ) and a "compound" user made up of the two fully cooperating sources (1 and 2) with power constraint $4 P$ and rate $2 R$. Since the two equivalent users in the new system are connected via finite-capacity links $C$, the capacity region of [1] can be adapted using standard arguments to a GMAC (see also [16]) to obtain the following upper bound:

$$
R_{\text {upper }}^{\prime \prime}=\sup _{\substack{f(u) f\left(\tilde{x}_{1} \mid u\right) f\left(\tilde{x}_{2} \mid u\right): \\
E\left[\tilde{X}_{1}^{2}\right] \leq 4 P, E\left[\tilde{X}_{2}^{2}\right] \leq P}} \min \left\{\begin{array}{l}
\frac{1}{2} I\left(\tilde{X}_{1} ; Y \mid \tilde{X}_{2} U\right)+\frac{C}{2}, \\
I\left(\tilde{X}_{2} ; Y \mid \tilde{X}_{1} U\right)+C, \\
\frac{1}{3} I\left(\tilde{X}_{1}, \tilde{X}_{2} ; Y \mid U\right)+\frac{2}{3} C, \\
\frac{1}{3} I\left(\tilde{X}_{1}, \tilde{X}_{2} ; Y\right)
\end{array}\right\},
$$

where variables $\tilde{X}_{1}$ and $\tilde{X}_{2}$ represent the inputs of the "compound" and "regular" users, respectively, and 
$f(u) f\left(\tilde{x}_{1} \mid u\right) f\left(\tilde{x}_{2} \mid u\right)$ factorizes the corresponding joint probability density function $f\left(u, \tilde{x}_{1}, \tilde{x}_{2}\right)$. This latter constraint is equivalent to the Markov chain condition $\tilde{X}_{1}-U-\tilde{X}_{2}$. What is now left to prove is that a joint Gaussian distribution $f(u) f\left(\tilde{x}_{1} \mid u\right) f\left(\tilde{x}_{2} \mid u\right)$ is optimal for (7), from which (6) would follow easily. This has been shown in [16].

Remark 2: It is easy to see that the upper bound (5) would hold also in the presence of undirected links with overall capacity $C$, whereas (6) would need to be modified by adding an optimization over the allocation of the conferencing capacity on the links between the "regular" and "compound" users (see Remark 1).

\section{An Achievable Rate}

In this section we derive an achievable equal rate and prove its optimality for large enough ring capacity $C$, thus establishing the capacity of the channel in Fig. 1 in such regime. Numerical results are then provided in Sec. V to get further insight into the properties of the derived achievable rate.

Proposition 2: The following equal rate is achievable for the system in Fig. 1:

$$
R=\min \left\{\frac{1}{6} \log \left(1+3 P_{p}+6 P_{c}+9 P_{a}\right), \frac{C}{2}+r\right\}
$$

with

$$
r=\frac{1}{2} \min \left\{\begin{array}{l}
\frac{1}{6} \log \left(\left(1+3 P_{p}+6 P_{c}\right)\left(1+3 P_{p}\right)\right), \\
\frac{1}{4} \log \left(\left(1+3 P_{p}+6 P_{c}\right)^{1 / 3}\left(1+3 P_{p}+2 P_{c}\right)\right), \\
\frac{1}{4} \log \left(\left(1+3 P_{p}+4 P_{c}\right)\left(1+3 P_{p}\right)^{1 / 3}\right), \\
\frac{1}{6} \log \left(\left(1+3 P_{p}+4 P_{c}\right)\left(1+3 P_{p}+2 P_{c}\right)\right),
\end{array}\right.
$$

and with the power constraint $P=P_{a}+P_{c}+P_{p}$.

Remark 3 (Sketch of proof of Proposition 2): We use a threelayer transmission scheme whereby each $j$ th user splits its local message as $W_{j}=\left[W_{p, j} W_{c, j} W_{a, j}\right]$ with corresponding rates $R=R_{p}+R_{c}+R_{a}\left(W_{b, j} \in\left\{1,2, \ldots, 2^{n R_{b}}\right\}\right.$ for $b=p, c, a)$. The private message $W_{p, j}$ is to be kept locally at the $j$ th user, whereas the two common messages $W_{c, j}$ and $W_{a, j}$ are exchanged over the ring as follows: The common message $W_{c, j}$ is intended only for the adjacent user $[j+1]$, whereas $W_{a, j}$ is destined to all the other two users. The common messages are sent over the ring in $k=2$ conferencing rounds so that, at the end of the conferencing phase, each user has available its common message $W_{p, j}$, the "pair-wise" common messages $W_{c, j}$ and $W_{c,[j-1]}$ and the "network-wise" common messages $W_{a, 1}, W_{a, 2}$ and $W_{a, 3}$. In the transmission phase, the users exploit the availability of common messages by creating correlated inputs, following the strategy of [14]. The powers $P_{p}, P_{c}$ and $P_{a}$ in (8) are used by each user to transmit the private part, the pair-wise common parts and the network-wise common parts, respectively. The details of the proof are worked out in Appendix-B.

By comparing the achievable rate (8) with the upper bound of Proposition 1, we get the following result.

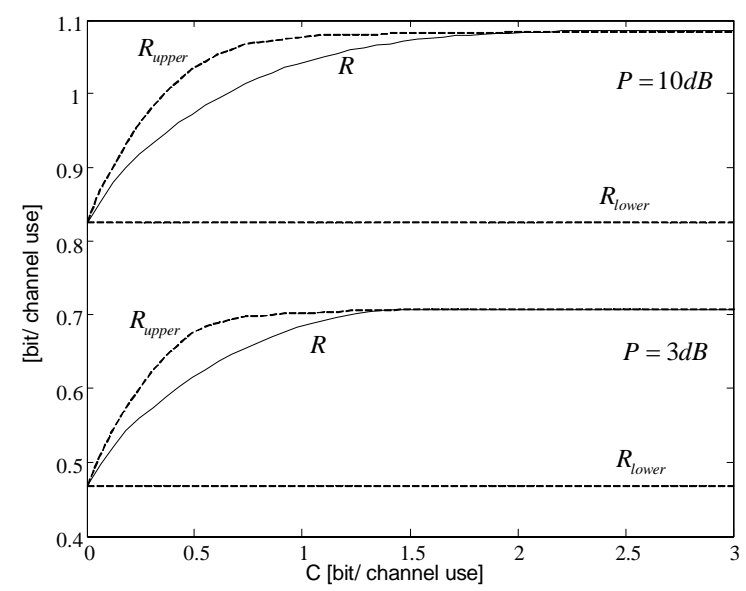

Fig. 2. Achievable rate $R$ ( 8) and upper bound (5), along with the noncooperative rate $R_{\text {lower }}=1 / 6 \cdot \log (1+3 P)$, for $P=3$ and $10 \mathrm{~dB}$ with optimal power allocation (evaluated numerically).

Proposition 3: If

$$
\frac{C}{2} \geq \frac{1}{6} \log (1+9 P),
$$

than the achievable rate derived in Proposition 2 is the capacity of the system in Fig. 1 and is equal to $R=1 / 6 \cdot \log (1+9 P)$.

Proof: The result follows by setting the power allocation to $P_{a}=P$ in (8) and comparing the corresponding achievable rate with the upper bound (5).

Remark 4: From standard results on the (non-cooperative) GMAC, for $C=0$, the capacity of the system is $1 / 6 \cdot \log (1+$ $3 P$ ), which is attained by using the strategy described above with power allocation $P_{p}=P$ (and it coincides with the upper bound (5)).

Remark 5: The rate (8) is clearly achievable also in the presence of undirected links by simply allocating all the ring capacity $C$ in the clockwise direction as in Fig. 1. However, it is not clear whether in general a multi-layer strategy similar to the one investigated here could be devised that effectively exploits possible bi-directionality of the ring links to improve the achievable rate. In the regime (10), identified by Proposition 3 , this is not the case due to Remark 2 .

\section{NUMERICAL RESUlts}

In this section, we discuss a numerical example to get further insight into the performance of the proposed multi-layer strategy. Of particular interest is to understand how the optimal power allocation among private and common messages in (8) depends on the operating point, and specifically on the ring capacity $C$. Fig. 2 shows the achievable rate $R$ (8) with optimal power allocation (evaluated numerically), the upper bound (5) and a lower bound $R_{\text {lower }}=1 / 6 \cdot \log (1+3 P)$ corresponding to the non-cooperative case (i.e., $C=0$ ) for per-user power constraints $P=3$ and $10 \mathrm{~dB}$. The corresponding optimal power allocation is shown in Fig. 3 for $P=3 \mathrm{~dB}$. It is interesting to notice that transmission of the first common 


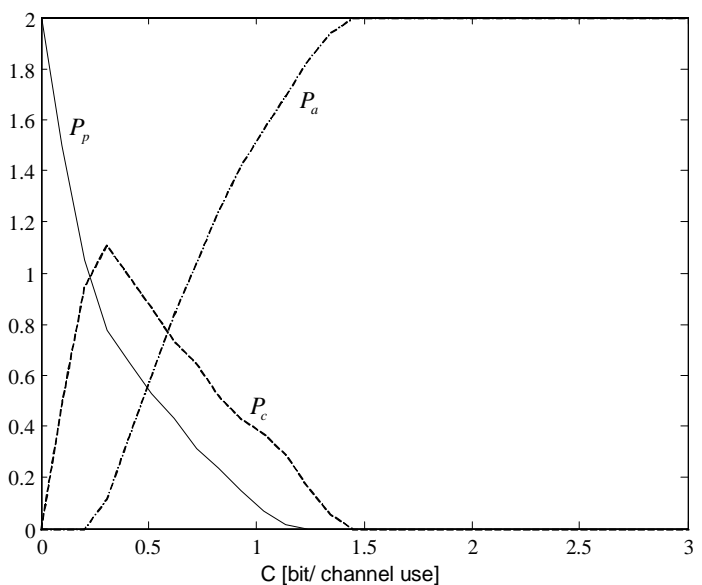

Fig. 3. Optimal power allocation between private message $\left(P_{p}\right)$, pairwise common messages $\left(P_{c}\right)$ and network-wise common messages $\left(P_{a}\right)$ that maximizes the achievable rate (8) for $P=3 \mathrm{~dB}$.

layer $\left(R_{c}\right)$, corresponding to pair-wise common messages, is useful for small-to-moderate values of $C$, while for larger values of $C$, as predicted by Proposition 3, transmitting only the network-wise common layer $\left(R_{a}\right)$ is the optimal strategy.

\section{CONCLUDING REMARKS}

This paper has studied an extension of the two-user MAC with conferencing encoders of [1] to the case of three users "conferencing” over a ring of uni-directional links. The analysis has focused on a Gaussian scenario and we have derived upper and lower bounds on the equal achievable rate, which meet whenever the ring capacity is large enough. The considered transmission strategy naturally extends the approach in [1] by considering a multi-layered transmission scheme based on the results in [14]. Optimality of the considered strategy for low-to-intermediate values of the ring capacity remains an open problem due to the difficulty in devising converse arguments that account for the nature of communications over the ring.

\section{APPENDIX}

\section{A. Appendix-A: Proof of Proposition 1}

We consider the derivation of the upper bound (5). Using Fano's inequality, imposing $P_{e} \leq \delta_{n}$ with $\delta_{n} \rightarrow 0$ for $n \rightarrow \infty$ leads to $n \varepsilon_{n} \geq H\left(W_{\{1,2,3\}} \mid Y^{n}\right) \geq H\left(W_{\{1,2,3\}} \mid Y^{n}, V_{\{1,2,3\}}^{k}\right)$, where $\varepsilon_{n} \rightarrow 0$ for $n \rightarrow \infty$ and the last inequality follows from the fact that conditioning reduces entropy. Therefore, we have

$$
\begin{aligned}
3 n R= & H\left(W_{\{1,2,3\}}\right) \\
= & H\left(W_{\{1,2,3\}} \mid Y^{n}, V_{\{1,2,3\}}^{k}\right)+I\left(W_{\{1,2,3\}} ; Y^{n}, V_{\{1,2,3\}}^{k}\right) \\
\leq & n \varepsilon_{n}+I\left(W_{\{1,2,3\}} ; V_{\{1,2,3\}}^{k}\right) \\
& +I\left(W_{\{1,2,3\}} ; Y^{n} \mid V_{\{1,2,3\}}^{k}\right),
\end{aligned}
$$

For the second term above, we can bound $I\left(W_{\{1,2,3\}} ; V_{\{1,2,3\}}^{k}\right) \leq H\left(V_{\{1,2,3\}}^{k}\right) \leq \sum_{i=1}^{3} H\left(V_{i}^{k}\right) \stackrel{(b)}{\leq} 3 n C$, from the definition of the constraints on the conferencing links (see Sec. II). As far as the third term is concerned, we can write, following standard arguments $I\left(W_{\{1,2,3\}} ; Y^{n} \mid V_{\{1,2,3\}}^{k}\right) \leq \sum_{i=1}^{n} I\left(X_{\{1,2,3\}, i} ; Y_{i} \mid V_{\{1,2,3\}}^{k}\right)$. We can then conclude that

$$
\begin{aligned}
3 n R & \leq 3 n C+\frac{1}{n} \sum_{i=1}^{n} I\left(X_{\{1,2,3\}, i} ; Y_{i} \mid V_{\{1,2,3\}}^{k}\right)+n \varepsilon_{n} \\
3 n R & \leq \sum_{i=1}^{n} I\left(X_{\{1,2,3\}, i} ; Y_{i}\right)+n \varepsilon_{n},
\end{aligned}
$$

where the second inequality follows by similar arguments. From the above inequalities, we get, respectively (dropping the $n \varepsilon_{n}$ terms for simplicity):

$$
3 R \leq 3 C+\frac{1}{2 n} \sum_{i=1}^{n} \log \left(1+3 \operatorname{var}\left(X_{j, i} \mid V_{\{1,2,3\}}^{k}\right)\right)
$$

and

$$
3 R \leq \frac{1}{2 n} \sum_{i=1}^{n} \log \left(1+E\left[\left(\sum_{j=1}^{3} X_{j, i}\right)^{2}\right]\right),
$$

where in (12) we have used the fact that, conditioned on the conferencing messages $V_{\{1,2,3\}}^{k}$, the input signals $X_{\{1,2,3\}, i}$ are independent since $X_{j}^{n}=g_{j}\left(W_{j}, V_{[j-1]}^{k}\right)$ and the maximum entropy theorem (along with the concavity of the log function), while (12) follows from the maximum entropy theorem. We then have by symmetry

$$
E\left[\left(\sum_{j=1}^{3} X_{j, i}\right)^{2}\right]=3 E\left[X_{j, i}^{2}\right]+6 E\left[X_{j, i} X_{j^{\prime}, i}\right],
$$

with $j^{\prime} \neq j$. Moreover, we can write (see [17])

$$
\begin{aligned}
E\left[X_{j, i} X_{j^{\prime}, i}\right]= & E_{V_{\{1,2,3\}}^{k}\left[E\left[X_{j, i} X_{j^{\prime}, i} \mid V_{\{1,2,3\}}^{k}\right]\right]}=E_{V_{\{1,2,3\}}^{k}\left[E\left[X_{j, i} \mid V_{\{1,2,3\}}^{k}\right] E\left[X_{j^{\prime}, i} \mid V_{\{1,2,3\}}^{k}\right]\right]} \\
\leq & \sqrt{E_{V_{\{1,2,3\}}^{k}\left[E\left[X_{j, i} \mid V_{\{1,2,3\}}^{k}\right]^{2}\right]}} \\
& \cdot \sqrt{E_{V_{\{1,2,3\}}^{k}\left[E\left[X_{j, i} \mid V_{\{1,2,3\}}^{k}\right]^{2}\right]}\left[X_{j, i}\right]-\operatorname{var}\left(X_{j, i} \mid V_{\{1,2,3\}}^{k}\right),}
\end{aligned}
$$

where in the last equality we have used the fact that $\operatorname{var}(A \mid B)=E\left[A^{2}\right]-E_{B}\left[E[A \mid B]^{2}\right]$ and the symmetry of the model. Finally, defining $E\left[X_{j, i}^{2}\right]=P_{i}, \operatorname{var}\left(X_{j, i} \mid V_{\{1,2,3\}}^{k}\right)=$ $P_{i}^{\prime}$ and $1 / n \sum_{i=1}^{n} P_{i}^{\prime}=P^{\prime}$ (dependence on $j$ is again dropped by symmetry), we have from (12):

$$
\begin{aligned}
R & \leq C+\frac{1}{6 n} \sum_{i=1}^{n} \log \left(1+3 P_{i}^{\prime}\right) \\
& \leq C+\frac{1}{6} \log \left(1+3 P^{\prime}\right),
\end{aligned}
$$


by the concavity of the $\log$ function, and similarly $R \leq$ $\frac{1}{6} \log \left(1+9 P-6 P^{\prime}\right)$. This concludes the proof.

\section{B. Appendix-B: Proof of Proposition 2}

In the conferencing phase, by using routing over the conferencing links, from flow considerations, the following condition is sufficient for exchanging pair-wise and networkwise common messages:

$$
C \geq R_{c}+2 R_{a} .
$$

Turning to the transmission phase, we exploit the rate region in Sec. VII of [14]. To do so, let us introduce four auxiliary random variables $Z_{c,\{1,2,3\}}, Z_{a}$, accounting respectively for the three pair-wise common messages $W_{c, j}$ and the networkwise common message $W_{a}=\left[W_{a,\{1,2,3\}}\right]$. Now, fix a joint pdf of the involved variables that factorizes as

$$
\begin{aligned}
& p_{a}\left(z_{a}\right) p_{c}\left(z_{c, 1}\right) p_{c}\left(z_{c, 2}\right) p_{c}\left(z_{c, 3}\right) p_{x \mid z}\left(x_{1} \mid z_{a}, z_{c, 1}, z_{c, 3}\right) \\
& p_{x \mid z}\left(x_{2} \mid z_{a}, z_{c, 2}, z_{c, 1}\right) p_{x \mid z}\left(x_{3} \mid z_{a}, z_{c, 3}, z_{c, 2}\right) p\left(y \mid x_{1}, x_{2}, x_{3}\right)
\end{aligned}
$$

where it is noted that we have introduced only three pdfs describing the input, namely $p_{a}\left(z_{a}\right), p_{c}\left(z_{c}\right)$ and $p_{x \mid z}\left(x \mid z_{a}, z_{c}, z_{c}^{\prime}\right)$. With this symmetric choice, the inequalities of the rate region of [14] reduce to

$$
\begin{aligned}
R_{p} \leq & \min \left\{\begin{array}{l}
I\left(X_{1} ; Y \mid Z_{a}, Z_{c,\{1,2,3\}}\right), \\
\frac{1}{2} I\left(X_{\{1,2\}} ; Y \mid Z_{a}, Z_{c,\{1,2,3\}}\right), \\
\frac{1}{3} I\left(X_{\{1,2,3\}} ; Y \mid Z_{a}, Z_{c,\{1,2,3\}}\right.
\end{array}\right) \\
& \stackrel{(a)}{=} \frac{1}{3} I\left(X_{\{1,2,3\}} ; Y \mid Z_{a}, Z_{c,\{1,2,3\}}\right) \\
3 R_{p}+R_{c} \leq & I\left(X_{\{1,2,3\}} ; Y \mid Z_{a}, Z_{c,\{1,2\}}\right) \\
3 R_{p}+2 R_{c} \leq & I\left(X_{\{1,2,3\}} ; Y \mid Z_{a}, Z_{c, 1}\right) \\
3 R_{p}+3 R_{c} \leq & I\left(X_{\{1,2,3\}} ; Y \mid Z_{a}\right) \\
3 R_{p}+3 R_{c}+3 R_{a} \leq & I\left(X_{\{1,2,3\}} ; Y\right)
\end{aligned}
$$

where (a) can be proved by exploiting the submodularity property of the set function $I\left(X_{\mathcal{S}} ; Y \mid Z_{a}, Z_{c,\{1,2,3\}}\right)$. Now, using Fourier-Motzkin elimination recalling (15) and $R \leq$ $R_{p}+R_{c}+R_{a}$, we have

$$
\begin{aligned}
R \leq & \frac{C}{2}+\frac{1}{6} I\left(X_{\{1,2,3\}} ; Y \mid Z_{a}\right) \\
& +\frac{1}{6} I\left(X_{\{1,2,3\}} ; Y \mid Z_{a}, Z_{c,\{1,2,3\}}\right) \\
R \leq & \frac{C}{2}+\frac{1}{12} I\left(X_{\{1,2,3\}} ; Y \mid Z_{a}\right) \\
& +\frac{1}{4} I\left(X_{\{1,2,3\}} ; Y \mid Z_{a}, Z_{c,\{1,2\}}\right) \\
R \leq & \frac{C}{2}+\frac{1}{4} I\left(X_{\{1,2,3\}} ; Y \mid Z_{a}, Z_{c, 1}\right) \\
& +\frac{1}{12} I\left(X_{\{1,2,3\}} ; Y \mid Z_{a}, Z_{c,\{1,2,3\}}\right) \\
R \leq & \frac{C}{2}+\frac{1}{6} I\left(X_{\{1,2,3\}} ; Y \mid Z_{a}, Z_{c, 1}\right) \\
& +\frac{1}{6} I\left(X_{\{1,2,3\}} ; Y \mid Z_{a}, Z_{c,\{1,2\}}\right) \\
R \leq & \frac{1}{3} I\left(X_{\{1,2,3\}} ; Y\right) .
\end{aligned}
$$

Finally, we set Gaussian pdfs for the auxiliary random variables and codebook: $p_{a}\left(z_{a}\right) \sim \mathcal{N}\left(0, P_{a}\right), p_{c}\left(z_{c}\right) \sim$ $\mathcal{N}\left(0, P_{c} / 2\right)$ and $\left.p_{x \mid z}\left(x \mid z_{a}, z_{c}, z_{c}^{\prime}\right) \sim \mathcal{N}\left(z_{a}+z_{c}+z_{c}^{\prime}, P_{p}\right)\right)$, obtaining (8).

\section{ACKNOWLEDGMENT}

This research was supported in part by a Marie Curie Outgoing International Fellowship and the NEWCOM++ network of excellence both within the 7th European Community Framework Programme, by the Israel Science Foundation and by the U.S. National Science Foundation under Grants CNS06-25637, CNS-06-26611, and ANI-03-38807.

\section{REFERENCES}

[1] F. Willems, "The discrete memoryless multiple access channel with partially cooperating encoders," IEEE Trans. Inform. Theory, pp. 441445, vol. 29, no. 3, May 1983.

[2] U. Black, Optical Networks: Third Generation Transport Systems, Prentice Hall, Upper Saddle River, 2002.

[3] I. Maric, R. Yates, and G. Kramer, "Capacity of interference channels with partial transmitter cooperation," IEEE Trans. Inform. Theory, vol. 53, no. 10, pp. 3536-3548, Oct. 2007.

[4] A. H. Kaspi and T. Berger, "Rate-distortion for correlated sources with partially separated encoders," IEEE Trans. Inform. Theory, vol. IT-28, no. 6, pp. 828-840, Nov. 1982.

[5] J. Barros and S. D. Servetto, “The sensor reachback problem,” submitted to IEEE Trans. Inform. Theory.

[6] H. Behroozi and M. R. Soleymani, "Cooperative source coding for the two-terminal Gaussian CEO problem," in Proc. Vehicular Technology Conference (VTC-2006 Fall), pp. 1-5, Montréal, Canada, Sep. 25-28, 2006.

[7] Y. Oohama, "Universal coding for correlated sources with linked encoders," IEEE Trans. Inform. Theory, vol. 42, no. 3, pp. 837-847, May 1996.

[8] S. Jaggi and M. Effros, "Universal linked multiple access source codes," in Proc. IEEE International Symposium on Inform. Theory (ISIT 2002), pp. 95, Lausanne, Switzerland, Jun. 30- Jul. 5, 2002.

[9] C. T. K. Ng, I. Maric, A. J. Goldsmith, S. Shamai, and R. D. Yates, "Iterative and one-shot conferencing in relay channels," in Proc. IEEE Information Theory Workshop (ITW 2006), Punta del Este, Uruguay, Mar. 13-17, 2006.

[10] R. Dabora and S. Servetto, "Broadcast channels with cooperating decoders," IEEE Trans. Inform. Theory, vol. 52, no.12, pp. 5438-5454, Dec. 2006.

[11] S. C. Draper, B. J. Frey and F. R. Kschischang, "Interactive decoding of a broadcast message," Proc. Forty-First Annual Allerton Conference on Communication, Control, and Computing, Monticello, IL, 2003.

[12] S. M. Yazdi, S. Savari, K. Carlson and G. Kramer, "The capacity region of a collection of multicast sessions in an undirected ring network," in Proc. IEEE International Conference on Parallel Processing Workshops (ICPPW 2007), Xi'an, China, Sep. 10-14, 2007.

[13] S. A. Savari and G. Kramer, "The multimessage unicast capacity region for bidirectional ring networks," in Proc. IEEE International Symposium Inform. Theory, Seattle, WA, pp. 763-767, Jul. 2006.

[14] D. Slepian and J.K. Wolf, "A coding theorem for multiple access channels with correlated sources," Bell Systems Tech. J., vol. 52, pp. 1037-1076, Sep. 1973.

[15] T. Cover and J. Thomas, Elements of Information Theory, John Wiley \& Sons, Inc., New-York, 2006.

[16] S. I. Bross, A. Lapidoth and M. A. Wigger, "The Gaussian MAC with conferencing encoders," in Proc. IEEE International Symposium Inform. Theory (ISIT 2008), Toronto, Canada, Jul. 6-11, 2008.

[17] M. Wigger, Cooperation on the multiple-access channel, Ph.D. thesis, ETH, Zurich, 2008. 